\title{
A Case of Trimethoprim-Sulfamethoxazole-induced Hepatitis
}

\section{Oluyemisi Amoda*, MBBS, MHA, MPH, CPH, Elmarie Alexander, MD, Ese Uwagbale, MD}

Internal Medicine, Brookdale University Hospital and Medical center, 1 Brookdale Plaza, Brooklyn NY 11212, USA

*Corresponding Author: Oluyemisi Amoda, Internal Medicine, Brookdale University Hospital and Medical center,1 Brookdale Plaza, Brooklyn NY 11212, USA

Received date: 05 June 2021; Accepted date: 12 June 2021; Published date: 14 June 2021

Citation: Amoda O, Alexander E, Uwagbale E. A Case of Trimethoprim-Sulfamethoxazole-induced Hepatitis. J Med Case Rep Case Series 2(4): https://doi.org/10.38207/jmcrcs20210062

Copyright: (C) 2021 Oluyemisi Amoda. This is an open-access article distributed under the terms of the Creative Commons Attribution License, which permits unrestricted use, distribution, and reproduction in any medium, provided the original author and source are credited.

Abstract
A variety of pharmacologic agents are implicated in drug-induced hepatitis, the most common of which is Acetaminophen. However, there is
limited data regarding the incidence of Trimethoprim-Sulfamethoxazole (TMP-SMX) causing hepatitis. The objective of this case report is to
highlight the importance of TMP-SMX causing hepatitis. We present a case of an elderly female who developed hepatitis as evidenced by elevated
liver transaminases following the use of TMP-SMX for the treatment of urinary tract infection.

\section{Introduction}

Trimethoprim-Sulfamethoxazole (TMP-SMX) is a popular antibiotic used to treat urinary tract infections, pulmonary infections, and skin infections? It is a broad-spectrum antibiotic with activity against a lot of Gram-positive aerobic, Gram-negative aerobic, anaerobic, and atypical bacteria [1]. The commonly associated adverse effects noted with its use include hypersensitivity reactions, gastrointestinal

\section{Case Presentation}

An eight-year-old female with a history of hypertension, hyperlipidemia, and diabetes mellitus was admitted for left hip hemiarthroplasty after sustaining a left femoral neck fracture from a fall. A few days following discharge, she presented to the emergency department with jaundice and abdominal pain, both of one-day duration. The abdominal pain was localized to the right upper quadrant and mid-epigastrium regions, mild, intermittent, and relieved by analgesics. Of note, the patient had been diagnosed with Urinary Tract Infection (UTI) and was taking TMP-SMX for three days before she presented at the hospital. At presentation, she was symptoms such as nausea, vomiting, anorexia; skin reactions such as Stevens-Johnson syndrome, and Toxic Epidermal Necrolysis [2]. Hematological side effects such as anemia, agranulocytosis, thrombocytopenia, eosinophilia occur more rarely [1], [3]. However, rarely, ingestion may cause acute hepatitis, presenting with jaundice and abdominal pain.

hemodynamically stable and denied fever, nausea, vomiting, diarrhea, chest pain, pruritus, or dark-colored urine. Physical examination revealed jaundice and tenderness in the right upper and lower quadrants. Laboratory studies showed alanine aminotransferase (ALT) $376 \mathrm{U} / \mathrm{L}$, aspartate aminotransferase (AST) $665 \mathrm{U} / \mathrm{L}$, alkaline phosphatase (ALP) $1351 \mathrm{U} / \mathrm{L}$, Creatinine $2.77 \mathrm{mg} / \mathrm{dL}$, as shown in table 1. CT abdomen/pelvis and abdominal ultrasound showed ductal dilatation, gallstones with mild wall thickening without evidence of cholecystitis, as depicted in Figure 1. The pancreas, spleen, andliver appeared normal.

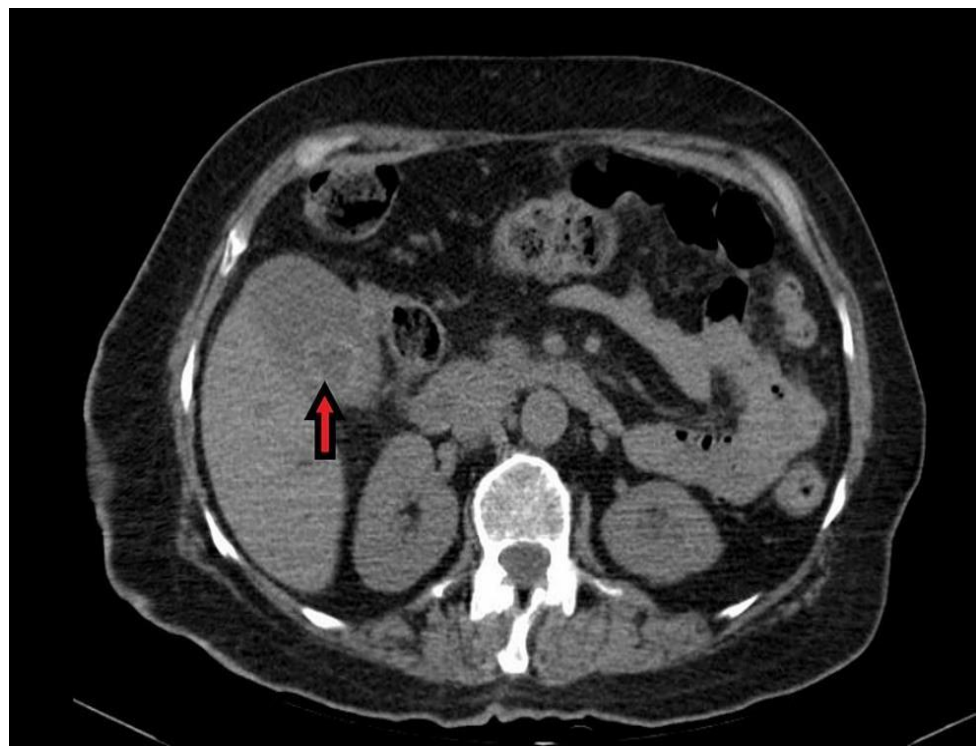

Figure 1: Computed Tomography of abdomen revealing dilated gallbladder with gallstones. 
Based on the timing of the TMP-SMX use and the development of jaundice three days after starting the medication, TMP-SMX was discontinued. Her treatment team changed her antibiotic regimen for UTI. She had Magnetic Resonance Cholangiopancreatography was done, which showed a significant oval $2.5 \mathrm{~cm}$ intraluminal filling defect compatible with gallstone, no common bile duct (CBD) dilatation, no apparent filling defect in $\mathrm{D}$, moderate $0.4-0.5 \mathrm{~cm}$ dilatation of the pancreatic duct, and fatty infiltration of the liver as seen in Figure 2.

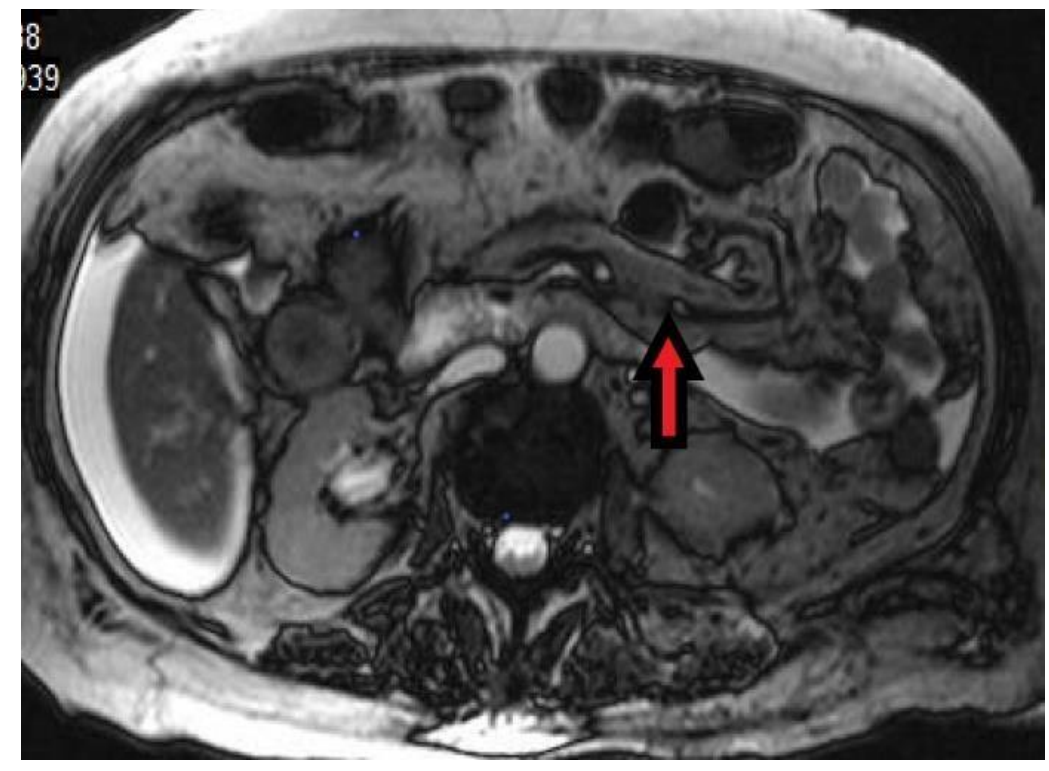

Figure 2: Magnetic Resonance Cholangiopancreatography showing moderate dilatation of the pancreatic duct.

She was initially managed for possible cholangitis with obstructive jaundice. Autoimmune workup was done, which was negative for anti-smooth antibodies, and mitochondrial antibodies; however, the antinuclear antibody screen was positive. After discontinuation of Bactrim, AST and ALT started trending down, but Alkaline phosphatase was still elevated, as showed in table 1.

Table 1: Liver enzymes at baseline, on presentation, and at discharge

\begin{tabular}{|l|l|l|l|}
\hline & Baseline Values & At Present & On discharge \\
\hline Alanine transaminase (ALT) & 79 & 356 & 17773 \\
\hline Aspartate transaminase (AST) & 73 & 665 & 381 \\
\hline Alkaline Phosphatase & 161 & 1351 & 1382 \\
\hline
\end{tabular}

N_Acetylcysteine protocol was initiated. The patient was later transferred out to a different facility for ERCP due to persistently elevated ALP.

\section{Discussion}

Drug-induced Hepatitis is defined as inflammation of the liver caused by certain medications toxic to the liver. Acute hepatic injury due to drugs has been reported in 5-10\% of patients hospitalized for jaundice. Drugs are the most common cause of fulminant hepatic failure in the US and Europe. The medication most associated with Drug-induced Hepatitis is Acetaminophen, commonly known as Tylenol. However, TMP/SMX has also been implicated in cases of drug-induced hepatitis. Our patient was evaluated for other causes of acute hepatitis, including viral infection, which was negative. She was also assessed for autoimmune hepatitis and primary biliary cirrhosis. In addition, results of her ERCP revealed possible cholangiocarcinoma, and she declined further workup, including biopsy, to confirm the diagnosis.

The pattern of hepatic injury can be cholestatic or mixed, with some courses more complicated and prolonged than others. It is proposed that the etiology of such damage is either due to a drug-allergy or metabolite-related toxicity [4]. In the latter mechanism, the hepatotoxic metabolite, hydroxylamine, is produced after TMP/SMX enters the metabolic pathway through cytochrome protein 450 (CYP450) subtype 2C9 in the liver [5].

The presence of elevated liver enzymes and jaundice has been associated with about 10-50 \% mortality in a rule known as Hy's law. The rule states that if both drug-induced hepatocellular injury (serum ALT and AST > 3 times the upper limits of normal) and jaundice (total bilirubin > two times the upper limit of normal) occur at the same time without biliary obstruction, mortality of at least $10 \%$ is expected. The sensitivity and specificity of clinical jaundice in patients with drug-induced hepatocellular injury are unknown. The most important predictors of outcome in drug-induced liver disease with hepatocellular injury have not been analyzed in many patients. Furthermore, information regarding the prognosis in other forms of drug-induced liver damage (e.g., DILD with cholestatic or mixed patterns) is limited [6]. The risk factors associated with developing drug-induced liver injury (DILI) include older age, female sex, obesity, alcohol use [7]. DILI can resolve spontaneously, progress to acute liver failure, hepatic necrosis, or result in death. Patients with 
already existing liver pathology or a history of DILI are more likely to have worse outcomes than those without [8]. Our patient is an elderly female, which puts her at an increased risk; however, she is

\section{Conclusions}

A few medications, including Acetaminophen, have been implicated in severe drug-induced liver injury. There has also been increasing incidence with many other drugs, including TMP/SMX. It could be argued that the rise in liver enzymes was caused by the cholangiocarcinoma but the elevation in AST and ALT developed after the medication was started and declined after it was stopped while the Alkaline Phosphatase remained persistently elevated. No doubt the cholangiocarcinoma could have contributed to the elevation

\section{References}

1. Wormser GP, Keusch GT, Heel RC (1982) Co-trimoxazole (trimethoprim-sulfamethoxazole): an updated review of its antibacterial activity and clinical efficacy. Drugs. 24(6): 459518.

2. Schöpf E (1987) Skin reactions to co-trimoxazole. Infection. 15(Suppl 5): S254-8.

3. Heimpel H, Raghavachar A (1987) Hematological side effects of co-trimoxazole. Infection. 15(Suppl 5): S248-53.

4. Björnsson E, Olsson R (2005) Outcome and prognostic markers in severe drug-induced liver disease. Hepatology. 42(2): 481-489.

5. National Institute of Diabetes and Digestive and Kidney Diseases. (2012: Sulfamethoxazole-Trimethoprim. In Liver not obese nor with pre-existing liver pathology.

At the time of discharge/transfer, her liver enzymes had already started trending down.

in liver enzymes, but it would not explain the decrease that occurred after the medication was stopped. Patients at increased risk of developing drug-induced liver injury includes the elderly, female sex, morbidly obese, persons with a pre-existing liver pathology, and chronic alcoholics. As a result, such factors should be identified when considering the antibiotic choice for patients, particularly in the elderly female, to assess alternatives to TMP/SMX.

ox: Clinical and Research Information on Drug-Induced Liver Injury [Internet].

6. Polson, J., \& Chalasani, N. (2008: Drug-induced liver disease (DILI). In Practical Management of Liver Diseases.

7. Regev A, Björnsson ES (2014) Drug-induced liver injury: morbidity, mortality, and Hy's law. Gastroenterology. 147(1): 20- 4 .

8. Yang JJ, Huang CH, Liu CE, Tang HJ, Yang CJ, et al. (2014) Multicenter study of trimethoprim/sulfamethoxazole-related hepatotoxicity: incidence and associated factors among HIVinfected patients treated for Pneumocystis jirovecii pneumonia. PLoS One. 9(9): e106141. 